\title{
Red de estructuración territorial histórica. El caso de la ruta de la Cíbola, en la época colonial
}

\section{Historic territorial estructuring road. The case of the route of the Cíbola, in the colonial period}

\author{
Pedro Gómez-Molina ${ }^{1}$ \\ Pedro S. Urquijo-Torres ${ }^{2}$ \\ Gerardo Bocco-Verdinelli ${ }^{3}$ \\ Universidad Nacional Autónoma de México, México
}

\begin{abstract}
Resumen
La llamada Ruta de Cíbola es el resultado de una serie de expediciones emprendidas desde el siglo XVI - aprovechando antiguos caminos y senderos prehispánicos-, que permitió la incursión y expansión colonial hacia el septentrión noroccidental novohispano. La ruta fue estudiada, entre otros, por Carl O. Sauer, dándola a conocer a nivel internacional. Nuestro propósito en esta ocasión es analizar cartográficamente este camino, así como las implicaciones territoriales que de él derivaron. Analizamos los cambios geográficos que se suscitaron con el avance fronterizo colonial. Es de resaltar que este camino de profundo raigambre es una de las vías que permitieron la expansión territorial novohispana por el noroccidente mexicano. Para ello, nos valemos de fuentes documentales y cartográficas primarias y su procesamiento a través de sistemas de información geográfica en su orientación histórica (SIG-H).
\end{abstract}

Palabras clave: estructuración territorial, Nueva España, caminos, frontera, septentrión.

1 Licenciado en Ciencias Ambientales, México, Escuela Nacional de Estudios Superiores, Universidad Nacional Autónoma de México, Campus Morelia, correo: pegomz014@gmail.com

2 Doctor en Geografía, México, Centro de Investigaciones en Geografía Ambiental, Universidad Nacional Autónoma de México, Campus Morelia, correo: psurquijo@ciga.unam-mx

3 Doctor en Geografía, México, Centro de Investigaciones en Geografía Ambiental, Universidad Nacional Autónoma de México, Campus Morelia, correo: gbocco@ciga.unam-mx

Este artículo corresponde a la ponencia presentada en el 35th Conference of Latin American Geographers realizada en San José, Costa Rica del 20 al 22 de mayo del 2018. 


\begin{abstract}
The Route of the Cibola arose from a series of expeditions undertaken beginning in the 16th Century - taking advantage of old roads and prehispanic trails - that allowed the incursion and expansion into the northwestern region of New Spain. The route was studied by, inter alia, Carl Sauer, who brought it international recognition. Our purpose here is to analyze this route cartographically and examine its territorial implications, which entails analysis of the geographical changes provoked concomitantly with the advance of the Colonial boundary. Th complementary, it analyzes the geographical changes that they provoked with de Colonial boundary advance. Notably, the old roads of the path was one of the factors facilitating the territorial expansion of New Mexico in the Mexican Northwest. For this study, we utilize primary document sources and process them through geographic information systems with a historical orientation (H-GIS).
\end{abstract}

Keywords: territorial estructuration, New Spain, roads, frontier, Septentrion.

\title{
Introducción
}

Este trabajo muestra el resultado de un estudio histórico-cartográfico donde se describe los cambios geográficos relacionados con la conformación de caminos. El caso a analizar es el de la llamada Ruta de la Cíbola, en la Nueva España entre los siglos XVI y XVII. De modo que se tiene que indagar caminos que permitieron la conformación de la ruta, y como estos fueron determinantes en la creación de una red de estructuración territorial en el periodo colonial novohispano.

La Ruta de la Cíbola es el resultado de un conjunto de expediciones, algunas de las más importantes y sobresalientes del siglo XVI, entre las que se cuentan las de Francisco de Cortés de Buenaventura (en 1524), Beltrán Nuño de Guzmán (1530), Diego Hurtado de Mendoza (1532), Diego de Guzmán (1533) o la célebre ruta de Álvar Núñez Cabeza de Vaca (1528-1536) (Figura 2). En diferentes momentos, los exploradores reutilizaron antiguos senderos indígenas como referencia inicial o retomaron estos para sus transectos (Sauer, 1998).

De acuerdo con Carl Sauer (1998), la ruta de la Cíbola era una vía que partía del Altiplano central mexicano, atravesaba las regiones costeras del noroeste novohispano y concluía en la California. Los indígenas septentrionales utilizaban los antiguos senderos para transportar turquesas hacia el sur y obtener plumas de aves de brillante plumaje de las tierras bajas subtropicales. También circulaban por esos caminos pieles de búfalo, conchas y perlas, metales y obsidiana. Ya como camino real colonial, la ruta permitía conectar las poblaciones, misiones, presidios, minas y ranchos del noroeste de la Nueva Galicia. 
Pedro Gómez-Molina, Pedro S. Urquijo-Torres, Gerardo Bocco-Verdinelli. Red de estructuración territorial histórica. El caso de la ruta de la Cíbola, en la época colonial

La investigación histórica sobre los caminos de la Nueva España han sido motivo de reflexión debido a la notable cantidad de cartografía histórica producto de los mismos, aunado a las relaciones comerciales y de comunicación entabladas en la época (Cramaussel, 2006). Lo que se pretende en este trabajo es ofrecer sintéticamente una visión de la vía noroeste, y de su revalorización como red de estructuración territorial.

En México, la cartografía histórica es uno de los campos de la geografía histórica con mayor tradición. Desde el siglo XIX, se han realizado investigaciones orientadas a los procesos de poblamiento e integración territorial, la expansión y contracción de fronteras y jurisdicciones civiles y eclesiásticas, el conocimiento toponímico, el aprovechamiento histórico de recursos naturales y el crecimiento de las ciudades, entre otros temas (Mendoza, 2000; Moncada 2003). En la actualidad, los mapas y documentos antiguos son insumos de investigación sumamente valiosos, susceptibles de analizarse a través de los SIG. En su orientación histórica, los SIG brindan la oportunidad de hacer correlaciones y asociaciones espaciales de los acontecimientos geográficos del pasado y proporcionan dinamismo a la cartografía histórica (Guzmán, 2017; Lefebvre 2017).

En el siglo XVI, tras el establecimiento de la Nueva España, los conquistadores europeos realizaron diferentes expediciones en la búsqueda de recursos minerales. A partir de ello, se escribieron crónicas, relatos de viaje, informes oficiales y se elaboraron mapas donde se narraba la apertura de caminos e incursiones de conquista, lo que significa en la actualidad una fuente de investigación histórica. En este sentido, existe un importante acervo documental, principalmente cartográfico, referente a la Ruta, el cual ha tenido poca atención geohistórica. Aprovechamos, entonces, estos insumos históricos para procesarlos a través de Sistemas de Información Geográfica (SIG). Ello permite una visualización general de la estructuración territorial de la Ruta, en la época colonial.

El SIG histórico hace referencia a una práctica metodológica orientada al análisis y procesamiento de cartográfica antigua, abriendo así nuevas posibilidades de análisis (Guzmán, 2017; Lefebvre 2017). Hay que precisar que el SIG histórico es una que herramienta que, si bien no resuelve todo lo que se pretende con la investigación, es una representación cartográfica que da mayor información espacial y que toma elementos de los mapas y su contexto histórico y geográfico. 


\section{Marco Metodológico}

Se procedió de la siguiente manera. En primer lugar, se realizó una extensa búsqueda bibliográfica sobre los elementos históricos del área de estudio, en el periodo comprendido entre los siglos XVI y XVII. Para lo cual se consideraron las crónicas de conquistadores y exploradores coloniales, tales como Álvar Nuñez Cabeza de Vaca (2000) o la información brindada por Hernán Cortés a través de sus Cartas de Relación. Asimismo, se consideró y procesó la amplia información que proporcionó Carl O. Sauer en 1938 (1998), en su estudio pormenorizado sobre la ruta de la Cíbola; el más importante que se haya realizado al respecto hasta la fecha.

Posteriormente, se realizó una búsqueda de cartografía histórica, que permitiera cotejar los cambios geográficos, expansión o diversificación del camino a través del tiempo. Para ello se realizó investigación de archivo en la Mapoteca Orozco y Berra y en el Archivo General de la Nación. Los mapas obtenidos fueron procesados a través de SIG (Software ArqGis versión 10.5 con licencia institucional). Se trabajó con dos mapas que datan de los siglos XVI y XVII, los cuales gradualmente disminuyen su precisión en cuanto más antiguos son. Estos documentos fueron elaborados con otras lógicas de representación del espacio, por lo que la georreferenciación requiere de estrategias particulares como el cotejar la información histórica con referencias actuales. Los datos contemporáneos fueron obtenidos a través de las bases de datos del Instituto Nacional de Estadística, Geografía e Informática (INEGI).

Para un mapa actual el error debe estar lo más cercano a cero, pero para el procesamiento de mapas históricos se consideró válido un valor menor o igual a tres. Posteriormente se seleccionaron puntos de control identificables en la cartografía histórica, tales como ríos, montañas, localidades o áreas costeras. Es importante subrayar que en esta fase se debe tener particular cuidado, ya que puntos actuales no necesariamente corresponden a los históricos, lo cual es muy común cuando nuestra referencia es una localidad. Por lo tanto, se rastreó y verificó en otras fuentes (documentos, crónicas, historia toponómica) los puntos de control.

El procesamiento anteriormente explicado permitió visualizar las transformaciones coloniales del camino noroeste novohispano. En este sentido, los mapas generales que ahora exponemos son el resultado preliminar y sintético de la correlación entre las crónicas y las cartografías. 
Pedro Gómez-Molina, Pedro S. Urquijo-Torres, Gerardo Bocco-Verdinelli. Red de estructuración territorial histórica. El caso de la ruta de la Cíbola, en la época colonial

\section{El camino del noroeste novohispano}

La Ruta de la Cíbola se estableció en el noroccidente de la Nueva España y hasta el suroeste de los actuales Estados Unidos de América (Figura 1). Su nombre derivó de la analogía realizada por los conquistadores europeos en el siglo XVI, entre el relato mitológico de las siete ciudades asiáticas de la abundancia de Cíbola y Quivira y los hallazgos en el territorio novohispano. Es importante señalar que la analogía no fue afortunada en los hechos, pues las siete ciudades americanas, resultaron ser sólo siete pequeños poblados de alrededor de quince o veinte millas de diámetro, siendo la más grande el poblado de Aztatlán. En la región, los conquistadores europeos encontraron que los indios usaban vestidos, cultivaban maíz, tenían sal y los hombres eran monógamos. Asimismo, grande fue su asombro cuando se percataron que no existía riqueza material alguna, ni oro, ni turquesas (Cué, 1994).

Explicada brevemente y de acuerdo a la investigación de Carl O. Sauer (1938), la ruta de la Cíbola, se estructuró de la siguiente manera. Los primeros trazos de se establecieron conforme a la avanzada del conquistador Francisco Cortés, alcalde de la villa de Colima, en el siglo XVI. El primer punto era el valle de Cihuatlán, ocupación original de Colima, y que hoy se conoce como Bahía de Navidad. Posteriormente se incursinaba en el valle de Espuchimilco, ahora conocido como de la Purificación en Xalipanga. La ruta continuó al este de Autlán, sobre el río Ayuquila, a la que se tiene registro como Valle de Milpa. Posteriormente estaba Ayutla. Finalmente, Francisco Cortés entrega en Etzatlán a dos compañeros, lo que es hoy la tierra baja de Tepic junto con la zona tropical. La Sierra Madre Occidental que es continua a la ruta tenía que evadirse para continuar su paso, siguiendo los altiplanos en lo que hoy es Acaponeta, Nayarit, Mazatlán, y atravesando los desiertos de Chihuahua y Arizona (Figura 1). 
Pedro Gómez-Molina, Pedro S. Urquijo-Torres, Gerardo Bocco-Verdinelli. Historic territorial estructuring road. The case of the route of the Cíbola, in the colonial period

Figura 1. Mapa de la ruta de la Cíbola.

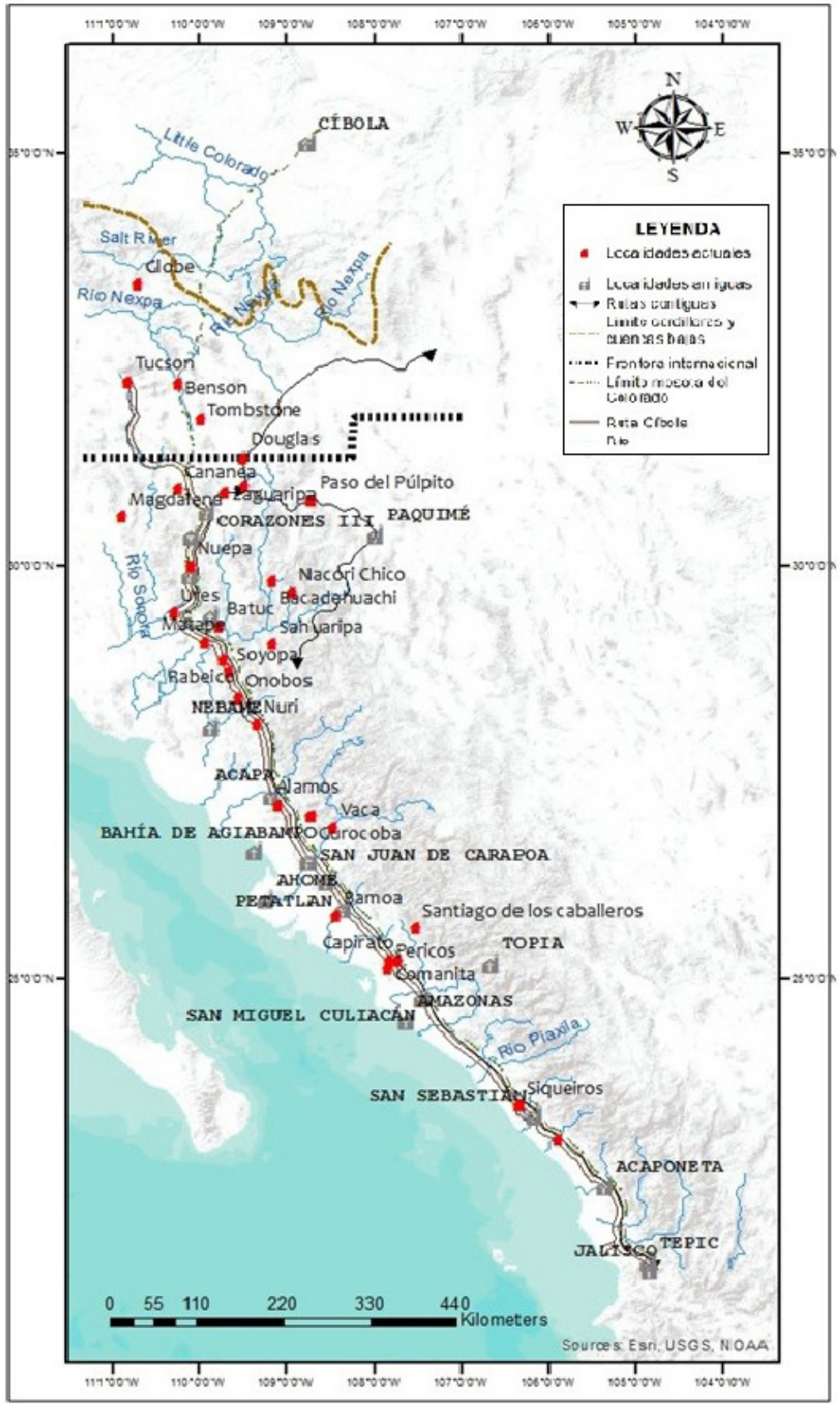

Fuente: Carl Sauer (1998). 
Pedro Gómez-Molina, Pedro S. Urquijo-Torres, Gerardo Bocco-Verdinelli. Red de estructuración territorial histórica. El caso de la ruta de la Cíbola, en la época colonial

Figura 2. Mapa de exploraciones en el noroeste novohispano.

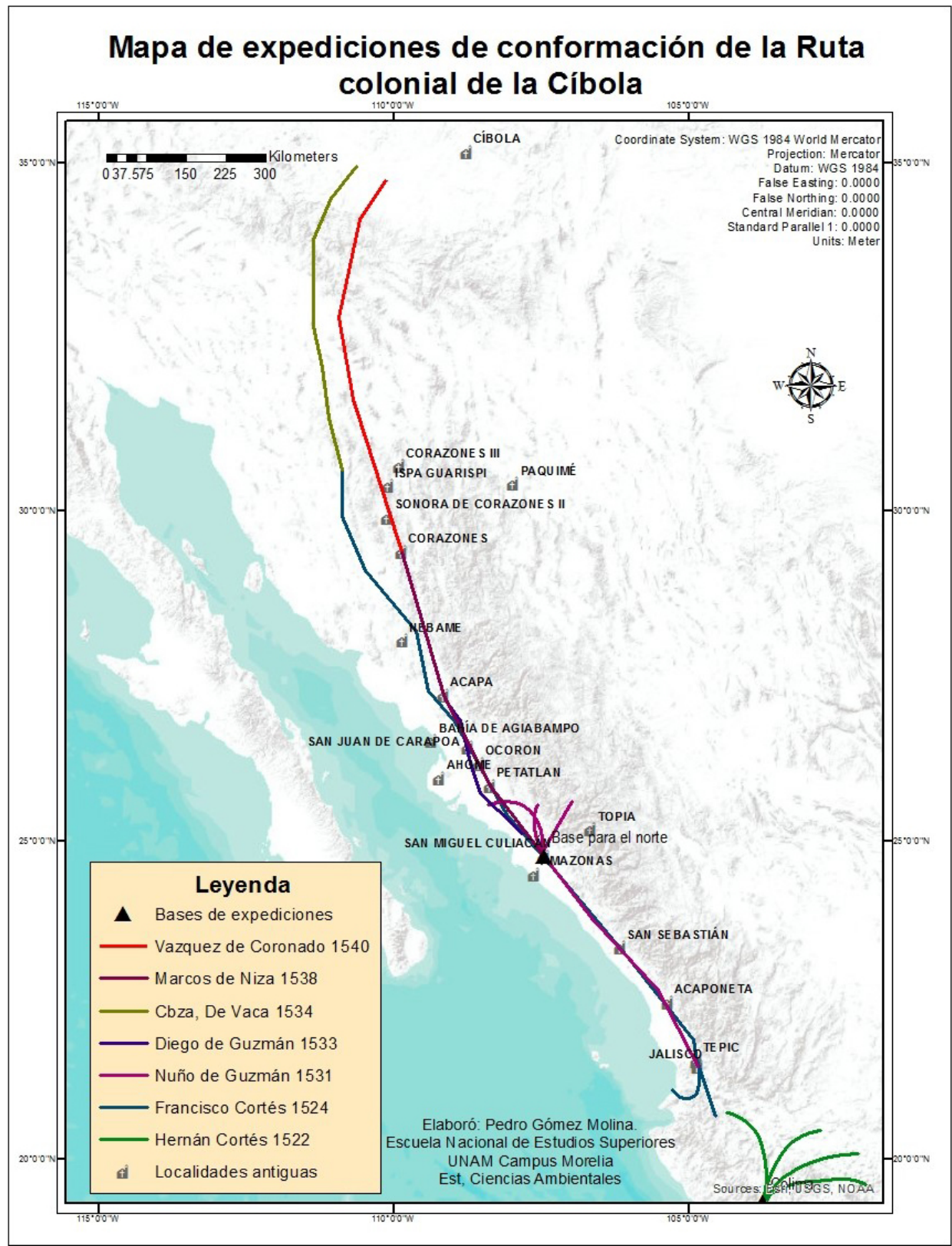

Fuente: Sauer ([1938], 1998). 
El camino fue parte de un sistema de red de estructuración territorial que se amplió, cruzando la Sierra Madre Occidental, en la medida que las expediciones de europeos y sus aliados indígenas se adentraban en el Septentrión. Los nodos territoriales que impulsaron las avanzadas españolas sobre la ruta fueron las localidades de Tepic, Acaponeta, Compostela y Colima, principalmente. Cabe mencionar que Colima ya tenía un gobierno establecido por Francisco Cortés -uno de los conquistadores encargados de las avanzadas españolas- (Sauer, 1972).

De Tepic al actual pueblo de Siqueiros, la flora nativa -que en aquél entonces era densa- complicó el paso de las incursiones de los colonizadores. Finalmente, de San Miguel Culiacán a los límites de la meseta del río Colorado, la expansión de la ruta no presentó mayores problemas para los conquistadores, ya que evitaron las grandes montañas de la Sierra, rodeándolas, y en el desierto no tuvieron complicaciones a su paso. Los mayores obstáculos a los que se enfrentaron eran las sequías y la falta de fuentes de abasto de agua, que resolvieron estableciendo contactos con los pobladores nativos del Septentrión. A partir de entonces, los extensos valles sirvieron a los colonizadores para la introducción de pastizales para la práctica ganadera. Además, la cercanía con el Océano Pacífico contribuyó al establecimiento de dinámicas económicas vinculadas a los puertos. Para la época, uno de los principales era el de San Blas, nodo de conexión marítima con Centro y Sur América.

En la Nueva España se instauró un modelo sistemático regional de "abasto indígena" para el territorio de Nueva Galicia - a donde pertenece la mitad de la Ruta de la Cíbola - y que perduró los primeros dos siglos de la época colonial. Este modelo se fundamentó en una política económica dirigida a la consolidación de un sistema colonial que estructuraba y habilitaba el espacio para la producción y abasto de alimentos, útil también para el proceso de colonización a corto, mediano y largo plazo. Este modelo regional de abasto indígena estuvo relacionado con corredores y arterias que constituyeron caminos coloniales (Rueda, 2009).

En el caso del occidente y noroeste novohispano, las autoridades coloniales abrieron un mercado regional en la provincia de Nueva Galicia - con salida a través del puerto de San Blas-, cuyas mercancías necesariamente transitaron por la Ruta de la Cíbola. De esta manera, tomaron 
Pedro Gómez-Molina, Pedro S. Urquijo-Torres, Gerardo Bocco-Verdinelli. Red de estructuración territorial histórica. El caso de la ruta de la Cíbola, en la época colonial

importancia estratégica y comercial las poblaciones de Mascota, Purificación y Sayula (Sauer, 1972; 1998).

A la par de los caminos establecidos por los colonizadores, los indígenas septentrionales continuaron desarrollando su propio comercio, a través del tránsito de alimentos y de mercancías, tales como la madera o barro. De esta manera lograron establecer corredores de abasto paralelos o vinculados a la ruta de la Cíbola, mediante una compleja red de senderos. Estas arterias territoriales entrelazaron las poblaciones indígenas sierra adentro con los caminos reales. El resultado de ello permitió a su vez conectar a los asentamientos indígenas y sus áreas de cultivo con los enclaves mineros, lo que favoreció que los excedentes agrícolas y frutícolas llegaran a los centros de explotación de minerales (Rueda, 2009). Para mostrar la diversificación de ramificaciones en la Ruta de la Cíbola se procesaron dos cartografías históricas que permiten visualizar, además de los caminos, los poblados y ríos principales: Mapa de zonas limitrofes entre las Audiencias de México y Nueva Galicia, siglo XVI (publicado en Calderón et al., 1984) y Mapa de las rutas y poblados en la Nueva Vizcaya, siglo XVII (Archivo General de la Nación, f. 2032) (Figura 3). 
Figura 3. Caminos históricos de la Ruta de la Cíbola.

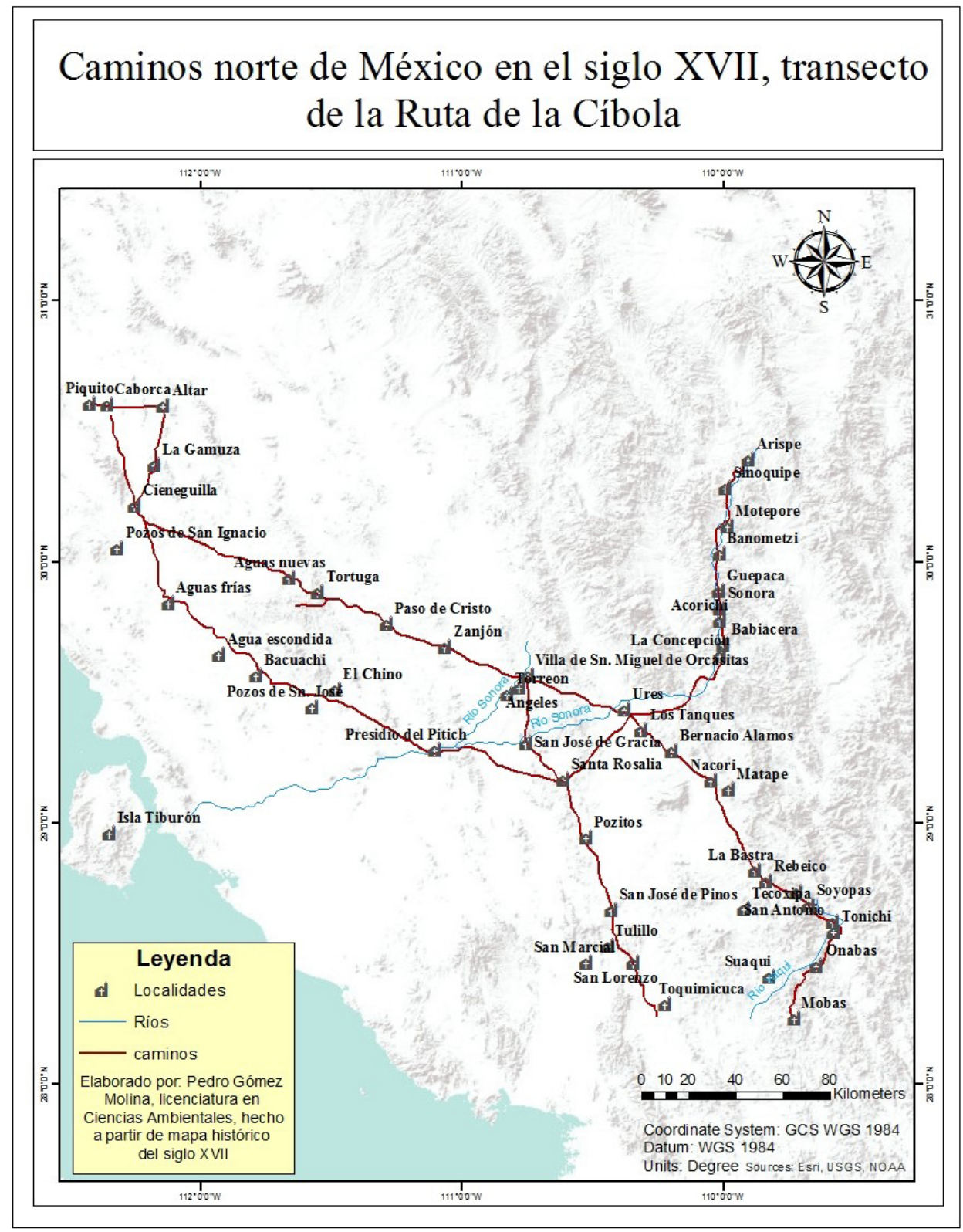

Fuentes Mapa de las zonas limítrofes entre las Audiencias de México y Nueva Galicia (Calderón, 1984); Rutas y poblados en la Nueva Vizcaya (Archivo General de la Nación), Sauer ([1938] 1998). 
Pedro Gómez-Molina, Pedro S. Urquijo-Torres, Gerardo Bocco-Verdinelli. Red de estructuración territorial histórica. El caso de la ruta de la Cíbola, en la época colonial

\section{Discusión}

La Ruta de la Cíbola fue una vía de estructuración principal que permitió conectar por el noroeste a las regiones novohispanas del centro y occidente con el Septentrión, hasta alcanzar la Alta California. Asimismo, dinamizó las interacciones económicas y culturales de las poblaciones de las actuales entidades mexicanas de Jalisco, Nayarit, Sinaloa, Sonora, Chihuahua y las estadounidenses de Arizona y California. Su importancia en los siglos XVI y XVII es notable; sin embargo, no ha captado la atención de más estudios contemporáneos. En este sentido, es importante subrayar la destacada labor emprendida por el geógrafo Carl O. Sauer, en el siglo $\mathrm{XX}$, quien dio difusión internacional al camino y es referente obligado.

El estudio de caminos antiguos mediante el uso de SIG en su orientación histórica permite, además, realizar estudios cartográficos comparativos y dinámicos. En el caso que ahora nos atañe, la Ruta de la Cíbola fue el origen de una serie de rutas de estructuración territorial que incluso en la actualidad siguen utilizándose o sirvieron de referencia. Al respecto, en la figura 4 mostramos cómo se visualiza ello (Figura 4). 
Pedro Gómez-Molina, Pedro S. Urquijo-Torres, Gerardo Bocco-Verdinelli. Historic territorial estructuring road. The case of the route of the Cíbola, in the colonial period

Figura 4. Mapa comparativo de la Ruta de la Cíbola y sus caminos y las actuales redes de estructuración territorial del noroeste mexicano.

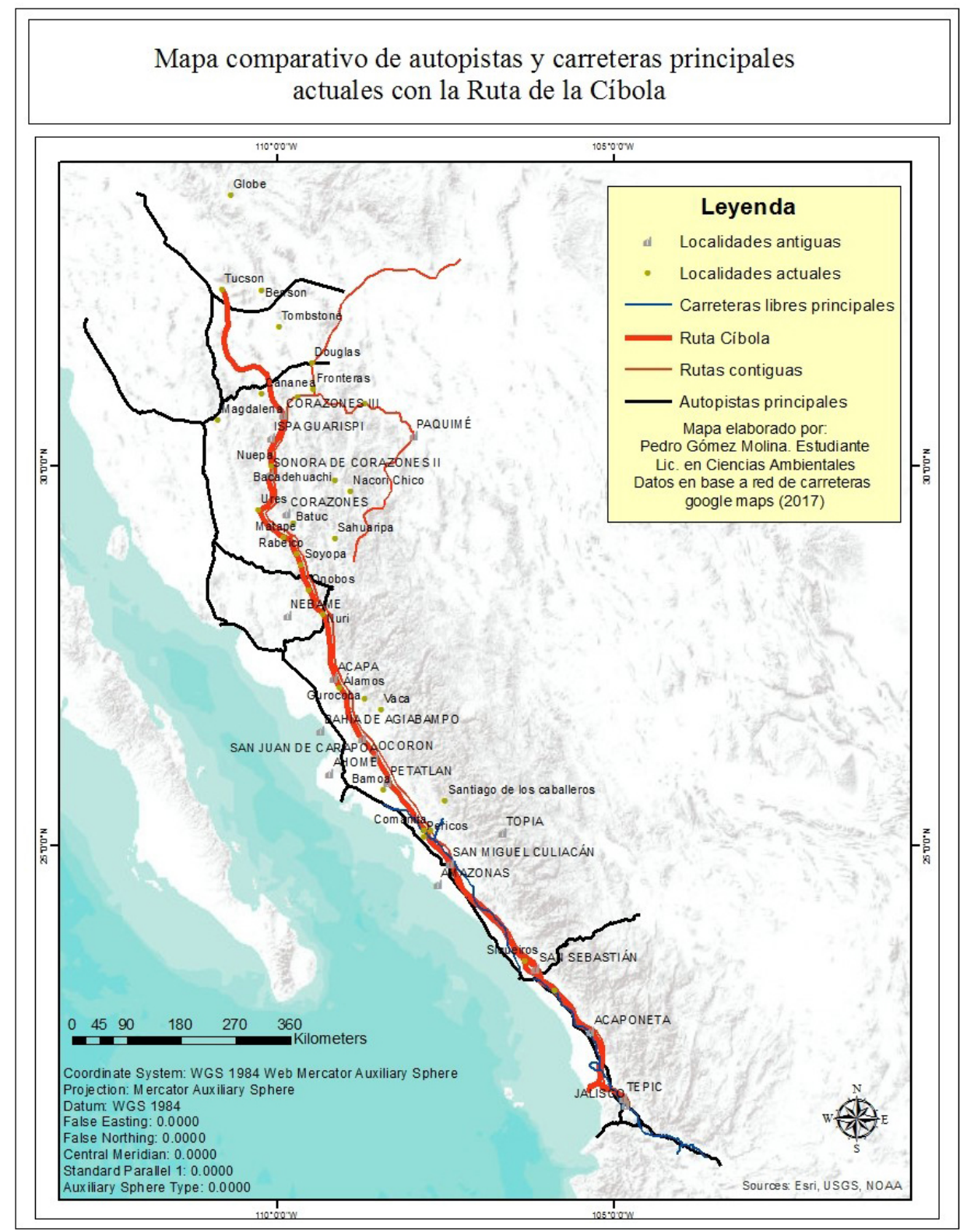

Fuente: Sauer ([1938] 1998); INEGI (2015). 
Pedro Gómez-Molina, Pedro S. Urquijo-Torres, Gerardo Bocco-Verdinelli. Red de estructuración territorial histórica. El caso de la ruta de la Cíbola, en la época colonial

Finalmente señalamos que el procesamiento de información documental y cartografía histórica mediante el uso de SIG, dinamiza la investigación sobre los lugares del pasado. Si bien estos insumos históricos no fueron elaborados para funcionar como fuentes de información, mediante estrategias de análisis, georreferenciación y contextualización es posible procesarlos y proyectarlos, mediante el uso de tecnologías geográficas contemporáneas. Lo que aquí hemos mostrado, es sólo un ejemplo preliminar y general de dicho proceso metodológico.

\section{Agradecimientos}

El texto fue elaborado en el marco del proyecto PAPIIT-DGAPA UNAM IA300817, "La Escuela Geográfica de Berkeley en México: aportaciones teóricas y metodológicas (1930-1960)". Los autores agradecen el financiamiento proporcionado para llevar a cabo esta investigación.

\section{Referencias}

Calderón, J. A. (ed.). (1984). Cartografía histórica de la Nueva Galicia, Guadalajara, Universidad de Guadalajara/Escuela de Estudios Hispano-Americanos.

Cramaussel, C. (2006). "Introducción”, en C. Cramaussel (coord.), Rutas de la Nueva España, Zamora, El Colegio de Michoacán, pp. 17-23.

Cué, M. E. (1994). "El mito de las siete ciudades", Anales de Antropología (31), pp. 167-211.

Del Bosque, I., C. Fernández, L. Martín-Forero, E. Pérez (2012). Los sistemas de información geográfica y la investigación en ciencias sociales y humanas. Madrid, Confederación Española de Centros de Estudios Locales (CSIC).

Guzmán, C. (2017). "Investigación histórica, los SIG y las nuevas posibilidades metodológicas y epistemológicas", en P.S. Urquijo, A. Vieyra y G. Bocco (coords.), Geografia e Historia Ambiental. Morelia, Centro de Investigaciones en Geografía Ambiental, UNAM, pp.193-214.

Lefebvre, K. (2017). “Colonialismo y paisaje, ¿cómo explotar los datos históricos para reconstruir el territorio colonial?, en P. S. Urquijo, A. Vieyra y G. Bocco (coords.), Geografía e Historia Ambiental, México, Centro de Investigaciones en Geografía Ambiental, UNAM, pp. 215-242. 
Mendoza, H. (2000). Los mapas y el siglo XX mexicano, en H. Mendoza (coord.), México a través de los mapas, México, Instituto de Geografía UNAM. 89-183.

Moncada, O. (2003). El nacimiento de una disciplina: la geografia en México (siglos XVI a XIX), México, Instituto de Geografía UNAM.

Nuñez Cabeza de Vaca, A. (2000). Naufragios, Buenos Aires, El Aleph.

Rueda, L. (2009). "Corredores de abasto indígena en la Nueva Galicia: un modelo regional de mercado. Sociedad y comercio colonial durante los siglos XVI y XVII", en J. Long Towell y A. Attolini Lecón (coords.), Caminos y mercados de México, México, Instituto de Investigaciones Históricas UNAM, pp. 327-348.

Sauer, C. O. ([1938] 1998). "La ruta de la Cíbola", en C. Sauer, Aztatlán, México, Siglo XXI, pp. 245-316.

Sauer, C. O. ([1945] 1976). Colima de la Nueva España en el siglo XVI, México, Editorial Jus. 